BMC

Genomics

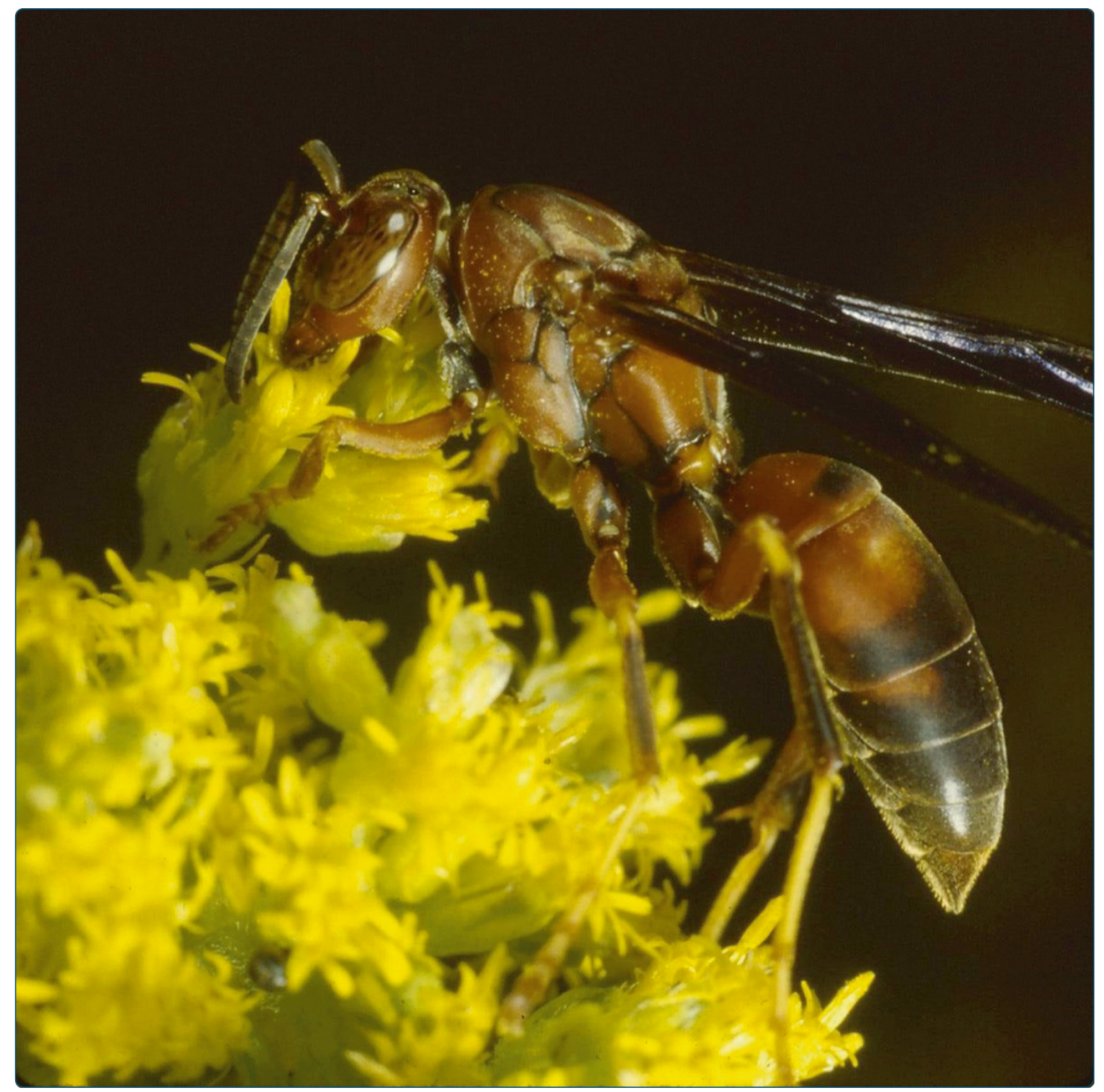

Shared genes related to aggression, rather than chemical communication, are associated with reproductive dominance in paper wasps (Polistes metricus)

Toth et al. 


\title{
Shared genes related to aggression, rather than chemical communication, are associated with reproductive dominance in paper wasps (Polistes metricus)
}

\author{
Amy L Toth ${ }^{1,2^{*}}$, John F Tooker ${ }^{3}$, Srihari Radhakrishnan ${ }^{1}$, Robert Minard ${ }^{4}$, Michael T Henshaw ${ }^{5}$ \\ and Christina M Grozinger ${ }^{3}$
}

\begin{abstract}
Background: In social groups, dominant individuals may socially inhibit reproduction of subordinates using aggressive interactions or, in the case of highly eusocial insects, pheromonal communication. It has been hypothesized these two modes of reproductive inhibition utilize conserved pathways. Here, we use a comparative framework to investigate the chemical and genomic underpinnings of reproductive dominance in the primitively eusocial wasp Polistes metricus. Our goals were to first characterize transcriptomic and chemical correlates of reproductive dominance and second, to test whether dominance-associated mechanisms in paper wasps overlapped with aggression or pheromone-related gene expression patterns in other species. To explore whether conserved molecular pathways relate to dominance, we compared wasp transcriptomic data to previous studies of gene expression associated with pheromonal communication and queen-worker differences in honey bees, and aggressive behavior in bees, Drosophila, and mice.
\end{abstract}

Results: By examining dominant and subordinate females from queen and worker castes in early and late season colonies, we found that cuticular hydrocarbon profiles and genome-wide patterns of brain gene expression were primarily associated with season/social environment rather than dominance status. In contrast, gene expression patterns in the ovaries were associated primarily with caste and ovary activation. Comparative analyses suggest genes identified as differentially expressed in wasp brains are not related to queen pheromonal communication or caste in bees, but were significantly more likely to be associated with aggression in other insects (bees, flies), and even a mammal (mice).

Conclusions: This study provides the first comprehensive chemical and molecular analysis of reproductive dominance in paper wasps. We found little evidence for a chemical basis for reproductive dominance in P. metricus, and our transcriptomic analyses suggest that different pathways regulate dominance in paper wasps and pheromone response in bees. Furthermore, there was a substantial impact of season/social environment on gene expression patterns, indicating the important role of external cues in shaping the molecular processes regulating behavior. Interestingly, genes associated with dominance in wasps were also associated with aggressive behavior in bees, solitary insects and mammals. Thus, genes involved in social regulation of reproduction in Polistes may have conserved functions associated with aggression in insects and other taxa.

Keywords: Wasps, Social behavior, Genomics, Aggression, Pheromones, Chemical communication

\footnotetext{
* Correspondence: amytoth@iastate.edu

'Department of Ecology, Evolution, and Organismal Biology, lowa State University, Ames, IA 50011, USA

${ }^{2}$ Department of Entomology, lowa State University, Ames, IA, USA

Full list of author information is available at the end of the article
}

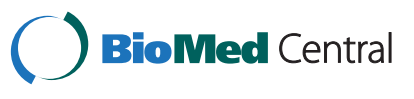

(c) 2014 Toth et al.; licensee BioMed Central Ltd. This is an Open Access article distributed under the terms of the Creative Commons Attribution License (http://creativecommons.org/licenses/by/2.0), which permits unrestricted use, distribution, and reproduction in any medium, provided the original work is properly credited. The Creative Commons Public Domain Dedication waiver (http://creativecommons.org/publicdomain/zero/1.0/) applies to the data made available in this article, unless otherwise stated. 


\section{Background}

In many animal species, social interactions with conspecifics can profoundly influence individual physiology and behavior, including reproduction [1]. Eusocial insect societies represent an extreme case in which colonies consist of one or a small number of reproductively active queens or kings and tens to millions of sterile workers. In some species, direct physical aggression establishes reproductive dominance, while other species use chemical signaling via pheromones to establish dominance hierarchies [2]. It has been hypothesized that such chemical communication systems evolved from an ancestral state in which aggressive dominance interactions inhibited reproduction $[3,4]$. Comparative studies of the genomic mechanisms mediating reproductive dominance through aggression or chemical signaling can determine if these modes of communication share common genetic underpinnings.

Comparisons of the genomic mechanisms underlying social regulation of reproductive dominance in primitively eusocial Polistes wasps and advanced eusocial Apis honey bees provide an excellent framework with which to study the evolution of social inhibition of worker reproduction [5]. Eusocial behavior evolved separately in bee and wasp lineages [6], and thus, any shared mechanisms for sociality and reproduction between honey bees and paper wasps may represent deeply conserved elements that could be employed in multiple insect lineages [5]. On one extreme, Polistes wasps use physical aggression to initially establish dominance hierarchies, and then transition to ritualized behaviors, possibly using chemically based recognition to maintain these hierarchies [7]. On the other extreme, honey bee queens use pheromones to establish reproductive dominance, and physical aggression by queens towards workers is not observed. The effects of honey bee queen pheromones on worker physiology, behavior, and gene expression patterns have been extensively characterized [8-10]. Conversely, gene expression associated with dominance status in Polistes has not been previously studied, but such work is now possible with the development of microarrays to monitor genome wide expression patterns in Polistes metricus wasps [11]. The goal of this study was to compare dominance-related gene expression in Polistes to aggression- and pheromone-related gene expression patterns in honey bees and other species, allowing us to test whether reproductive dominance in Polistes is associated with genes with known links to aggression, pheromonal regulation, or both.

The presence of distinct groups of females on Polistes colonies exhibiting a range of caste, dominance, and reproductive states [12] provides an excellent opportunity for dissecting the mechanistic bases of reproductive dominance. The fact that dominance hierarchies occur in different stages of colony development also allows us to examine the importance of the colony environment, which has proven to be very important in recent studies in other social insects [13]. In temperate species of Polistes, one or a few sister females found annual colonies in the spring "founding phase" [14]. If multiple females are present, these foundresses form a dominance hierarchy with dominant foundresses (with large ovaries) taking over egglaying and subordinate foundresses (with small ovaries) taking over foraging and provisioning of larvae [15]. During the "worker phase", foundress-reared brood emerge as adult females, which typically become workers [12]. At this time, the dominant foundress increases egg-laying and is called the queen, and subordinate foundresses either die or are forced from the nest [16,17]. Linear dominance hierarchy among the females characterizes worker phase nests, with the queen as the alpha, or most dominant, individual [15]. Among workers, both dominant workers (with partially developed ovaries) and subordinate workers (with mostly undeveloped ovaries) are socially inhibited from reproducing, but subordinates even more so due to receiving more aggressive contacts and engaging in energetically demanding foraging behavior [18-20].

The initial position of a Polistes individual in a dominance hierarchy is established within a few minutes via intense aggressive interactions with other females, including biting, grappling, and attempted stinging [21]. Since behavioral dominance is established rapidly, it is not likely to involve large-scale changes in gene expression or physiology, although prior physiological and hormonal state influences performance in dominance contests [22-25]. Subsequently, repeated, more ritualized dominance interactions maintain physiological, or reproductive, dominance [26]. Dominance hierarchy maintenance requires chemical and/or visual individual recognition between wasps [27]. Large physiological changes in ovary activation, juvenile hormone, and ecdysteroid titers accompany this longerterm reproductive dominance [28-30]. Here, we focus on reproductive (physiological) dominance rather than behavioral dominance, as this form of dominance is longer-term and thus more likely to be manifest at the level of gene expression.

This study focuses on transcriptomic and chemical correlates of reproductive dominance, both within and between the different female castes in Polistes metricus wasps. First, we examined chemical profiles in subordinate and dominant Polistes workers, nest-founding females (dominant and subordinate co-foundresses), and queens, to determine if there were chemical correlates of caste and reproductive dominance, as suggested by previous studies with other species of Polistes [31,32], which could potentially function as chemical signals or cues to establish or maintain reproductive dominance hierarchies. Next, we examined the gene expression profiles in the brains and ovaries of these five groups of wasps, to explore transcriptomic correlates of caste and reproductive dominance. 
The goals of the transcriptomic study were two-fold: first, to provide new baseline data on patterns of gene expression associated with dominance to identify candidate genes for future studies, and second, to conduct a comparative genomic analysis by quantitatively comparing wasp dominance-associated gene expression patterns to gene expression data in other species. We hypothesized that paper wasp brain gene expression patterns would be related to gene expression associated with caste, exposure to queen pheromone and/or aggression in honey bee workers. In addition, we extended our comparative analysis to available data on aggression-related gene expression from two non-eusocial species, the fruit fly Drosophila melanogaster and the mouse Mus musculus. The results of these studies suggest reproductive hierarchies in primitively social species are associated with gene networks related to aggression in solitary species rather than pheromonal regulation in advanced eusocial species.

\section{Results}

\section{Chemical analyses}

We examined chemical profiles from dominant and subordinate co-foundresses (sampled during the founding phase of the colony) as well as queens and dominant and subordinate workers (sampled during the worker-producing phase of the colony). We identified four body areas as candidate carriers of dominance-related chemicals: 1) cuticular hydrocarbons, previously associated with dominance status in $P$. dominula $[7,33,34]$; 2) the mandibular glands, where queen pheromone is produced in honey bees [8], 3) Dufour's glands because of their role in egg-marking in several social insect species [35], and 4) the sternal glands because of their potential importance to abdomen rubbing behavior which may accompany dominance interactions in Polistes [35]. Each of the four body regions examined had distinct chemical profiles, none of which were clearly related to dominance status. Data from the three glands are presented and discussed in the supplementary materials (Additional file 1: Supplemental Text, Figure S2).

From the cuticle, we identified 18 distinct hydrocarbons, a large proportion of which (13, or $72 \%$ ) showed significant differences across the five groups of wasps (Table 1). Linear discriminant analysis (LDA) revealed a separation between the foundresses and the queen/worker groups (Figure 1A); this could possibly reflect differences in season or social environment. Using GC-MS, we identified at least 16 different hydrocarbons of varying chain lengths from 25-40 (Table 1). Thirteen of these compounds were previously identified as present on the cuticle of Polistes metricus (Table 1, [36]). Hierarchical clustering of the 13 compounds with significant differences among the treatment groups (Figure 1B) revealed a correlated cluster of five compounds in the range of 33-40 carbon chain length (13,17- and 15,19-dimethyltritriacontane, 13-,15- and 17-

Table 1 Components of the cuticular hydrocarbons of Polistes metricus

\begin{tabular}{|c|c|c|c|c|}
\hline Putative ID & Retention time (min) & Carbon chain length & Molecular weight & p-value \\
\hline Pentacosane* & 27.6 & 25 & 352 & 0.004 \\
\hline n-octacosane* & 34.6 & 28 & 394 & 0.006 \\
\hline n-nonacosane* & 37.0 & 29 & 408 & 0.006 \\
\hline 11-, 13-, \& 15-methylnonacosane* & 37.6 & 30 & 422 & 0.03 \\
\hline n-triacontane* & 39.5 & 30 & 422 & 0.007 \\
\hline n-hentriacontane* & 41.1 & 31 & 436 & NS \\
\hline x-methyltriacontane (?) & 41.8 & 31 & 436 & 0.04 \\
\hline n-dotriacontane ${ }^{*}$ & 42.3 & 32 & 450 & NS \\
\hline $11,15-$ and $13,17-$ dimethylhentriacontane* & 45.0 & 33 & 464 & 0.004 \\
\hline 11,15- and 13,17-dimethylhentriacontane (?) * & 45.7 & 33 & 464 & NS \\
\hline $11-, 13-, 15-$ and 17-methyltritriacontane ${ }^{*}$ & 46.1 & 34 & 478 & NS \\
\hline Hexatriacontene isomer (?) & 47.4 & 36 & 504 & NS \\
\hline Hexatriacontene & 47.8 & 36 & 504 & 0.008 \\
\hline 13,17 - and 15,19-dimethyltritriacontane * & 49.4 & 35 & 492 & $<0.001$ \\
\hline 13-,15- and 17-methylpentatriacontane * & 49.9 & 36 & 506 & $<0.001$ \\
\hline 11,15- and 13,17-dimethylpentatriacontane * & 52.8 & 37 & 520 & 0.002 \\
\hline n-octatriacontane & 53.3 & 38 & 534 & $<0.001$ \\
\hline n-tetracontane & 56.7 & 40 & 562 & 0.03 \\
\hline
\end{tabular}

All identifications were high confidence (>90\% similarity to library or standards mass spectra) except for those indicated with (?). Compounds that differed in ANOVA analysis across the five groups are highlighted in bold and patterns presented graphically in Figure 1. Compounds with *were also identified as present in Polistes metricus in [36]. 

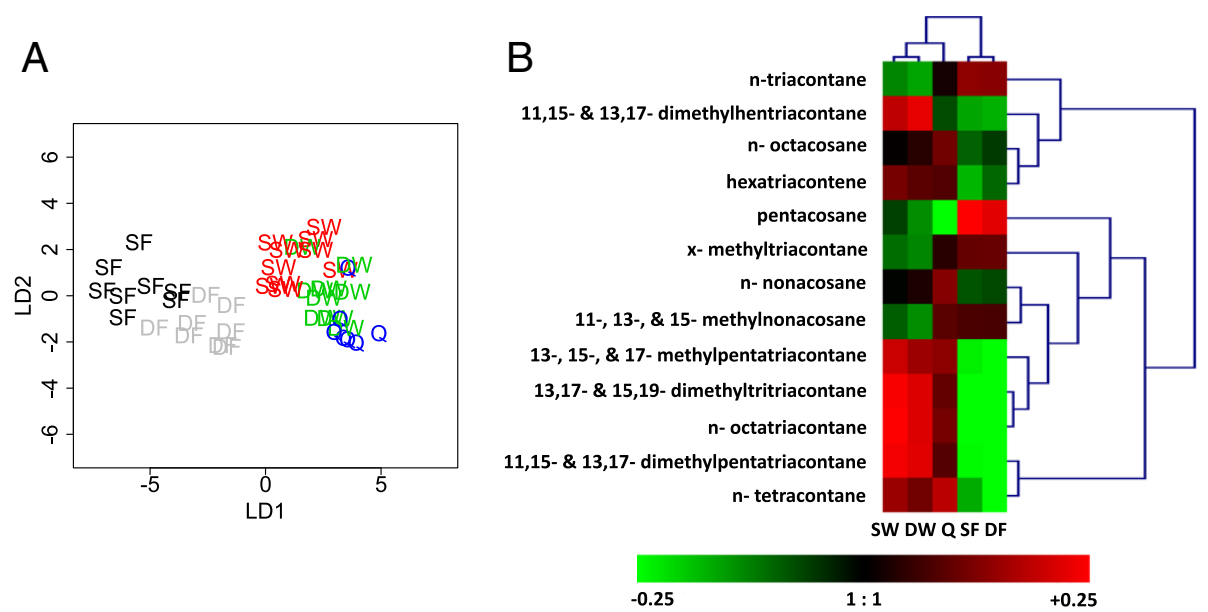

Figure 1 Multivariate analyses of cuticular hydrocarbon data. A) Linear discriminant analysis (LDA) of chemical profile data, showing graphs based on values of the two major linear discriminants, derived from quantities of compounds extracted from the cuticle from the five groups ( $D F=$ dominant foundress, SF = subordinate foundress, DW = dominant worker, SW = subordinate worker, $\mathrm{Q}=$ queen). B) Patterns of cuticular hydrocarbon abundance reveal a cluster of compounds related to season and/or social environment. Hierarchical clustering (represented by blue dendrograms) of mean values ( $\log _{10}$ transformed) for 13 compounds with significant differences across the five female groups. The heatmap illustrates the fold difference in $\log _{10}$ levels of each compound compared to the overall mean for each compound (1:1), with higher levels in red and lower in green. Five compounds (bottom of the figure) show a similar pattern in which levels are lowest in foundresses and highest in workers and queens, reflecting differences in season and/or social environment.

methylpentatriacontane, 11,15- and 13,19- dimethylpentatriacontane, n-octatriacontane, and n-tetracontane). All five compounds varied significantly among treatments (ANOVA, $\mathrm{P}<0.05$ ), and showed a similar pattern that reflected differences in season and/or social environment, with the highest levels in worker phase individuals (queens and workers) and lowest levels in founding phase individuals (foundresses).

\section{Brain gene expression}

Using previously developed custom oligo microarrays for $P$. metricus [11], we examined brain gene expression patterns of eight individuals from each of the five groups of wasps (dominant and subordinate co-foundresses, queens, and dominant and subordinate workers). Of the 5500 transcripts represented on the arrays, 3367 were expressed above background levels in a sufficient number of arrays to be included in the analysis. 499 of these (14.8\%) were differentially regulated across the five behavioral groups (FDR p-value <0.05).

Differentially regulated transcripts showed multiple distinct expression patterns across the five groups (multivariate analyses: Additional file 1: Figure S3). As in the case of the cuticular hydrocarbon profiles, there are clear differences associated with the two colony developmental phases. This effect of season/social environment on brain expression patterns was apparent in both hierarchical clustering based on a distance matrix of all possible contrasts (Figure 2A, Additional file 1: Figure S3C) and principal components analysis (PCA, Additional file 1: Figure S3B), where season/social environment accounted for 23\% of the overall expression variation.

Post-hoc contrasts across the groups (FDR p-value < 0.05 ) revealed relatively few transcripts were associated with dominance status. We focused on two contrasts (dominant vs subordinate foundresses, and dominant vs subordinate workers) because these contrasts represented females that were interacting together on the same nest and were not confounded by comparisons across castes. There were 46 differentially regulated transcripts between dominant and subordinate foundresses and 17 differentially regulated transcripts between dominant workers and subordinate workers. There was no overlap across these two contrasts, suggesting again a potent influence of season or social environment in that different mechanisms appear to be associated with dominance in foundresses and workers (Figure 2B). The 63 transcripts showing differences between dominant and subordinate females (the union of the aforementioned two contrasts) are heretofore referred to as "brain dominance-associated" transcripts (Figure 2B, Additional file 2).

Similarly, to identify caste-associated genes, we examined overlapping sets of genes from queen vs worker post-hoc contrasts (FDR p-value $<0.05$ ) and found a somewhat larger signal of differential expression. There were 118 differentially regulated transcripts between queens and dominant workers and 55 between queens and subordinate workers, 36 of which overlapped between the two contrasts (Figure 2B). The 137 transcripts showing differences between queens and workers (the 


\section{A}

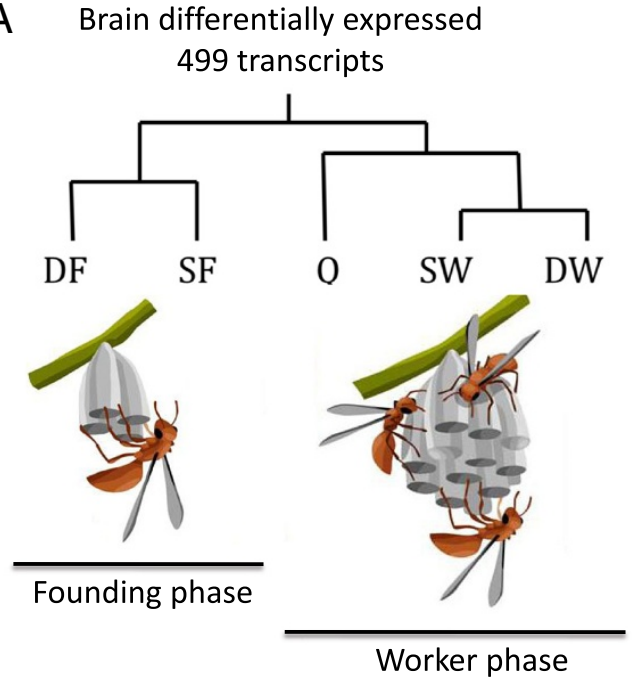

B
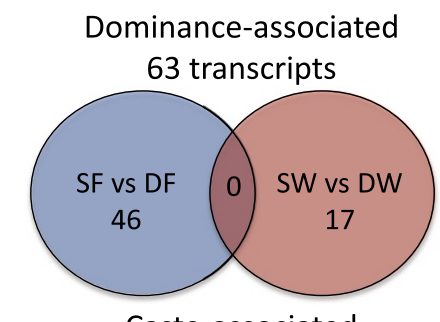

Caste-associated

137 transcripts

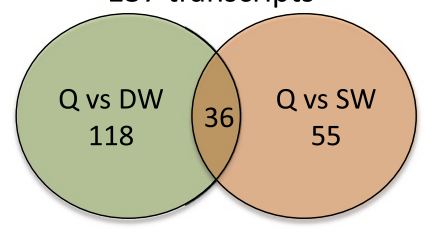

Figure 2 Patterns of gene expression in brains of dominant and subordinate wasps. A summary of the brain microarray data, for 499 differentially regulated transcripts across the five groups (DF = dominant foundress, SF = subordinate foundress, DW = dominant worker, SW = subordinate worker, Q = queen). A) Consensus clustering analysis (from both principal components analysis and hierarchical clustering) shows that many transcripts showed a pattern that corresponds to the social environment (founding phase or worker phase) and/or season. Wasp nest cartoons adapted from [37]. B) Venn diagrams summarizing the number of differentially regulated transcripts associated with either dominance status (top) or caste (bottom) and showing the overlaps between contrasts used to identify 'brain dominance-associated' transcripts (top) and 'brain caste-associated' transcripts (bottom).

union of the aforementioned two contrasts) are heretofore referred to as "brain caste-associated" transcripts (Figure 2B, Additional file 2).

We validated array expression data for one gene, vitellogenin, which was previously examined in queens and subordinate workers using quantitative real time PCR (qRT-PCR) [38]. Expression patterns uncovered with the array in the current study showed strikingly similar patterns to previous qRT-PCR data, with approximately 2-fold higher expression in queens compared to subordinate workers in both studies (Additional file 1: Figure S5).

\section{Ovary gene expression}

Next, we examined ovary gene expression patterns of eight individuals from each of the five groups of wasps (dominant and subordinate co-foundresses, queens, and dominant and subordinate workers). Out of 5500 transcripts represented on the array, 3349 were expressed above background levels in a sufficient number of arrays to be included in the analysis. Of those, we found a large proportion (2302, or $68.7 \%$ of transcripts) were differentially regulated across the five groups (after correcting for multiple testing, false discovery rate $\mathrm{p}$-value $<0.01$ ).

Again, there was a diversity of expression patterns across the five groups (multivariate analyses Additional file 1: Figure S4A, B, C). One of the most prevalent expression patterns (Figure 3A) reflects the gross level of ovary activation; queens and dominant foundresses had very high levels of ovary activation (score of 4 ) and were likely to be actively egg-laying, whereas the other three groups had very low ovary activation (scores of 1-2, and rarely 3, even in "dominant" workers) with no mature oocytes and were thus not actively egg-laying. This pattern was recovered both by hierarchical clustering by gene (Figure 3A, Additional file 1: Figure S4A) and PCA (Additional file 1: Figure S4B), in which ovary activation levels accounted for $41 \%$ of the variation.

Post-hoc contrasts across the groups (FDR p-value $<0.01$ ) revealed a moderately large number of transcripts were associated with dominance status. There were 657 differentially regulated transcripts between dominant and subordinate foundresses and 572 differentially regulated transcripts between dominant workers and subordinate workers. There was an overlap of 169 transcripts across these two contrasts (Figure 3B), suggesting that there may be both shared and divergent mechanisms associated with ovary activation across the reproductive and worker castes. The 1060 transcripts showing differences between dominant and subordinate females (the union of the aforementioned two contrasts) are heretofore referred to as "ovary dominance-associated" transcripts (Additional file 3).

By examining overlapping sets of genes from queen vs worker post-hoc contrasts (FDR p-value $<0.01$ ), we again found a larger signal of differential expression associated with caste differences. There were 1678 differentially regulated transcripts between queens and dominant workers and 1266 between queens and subordinate workers, 1001 


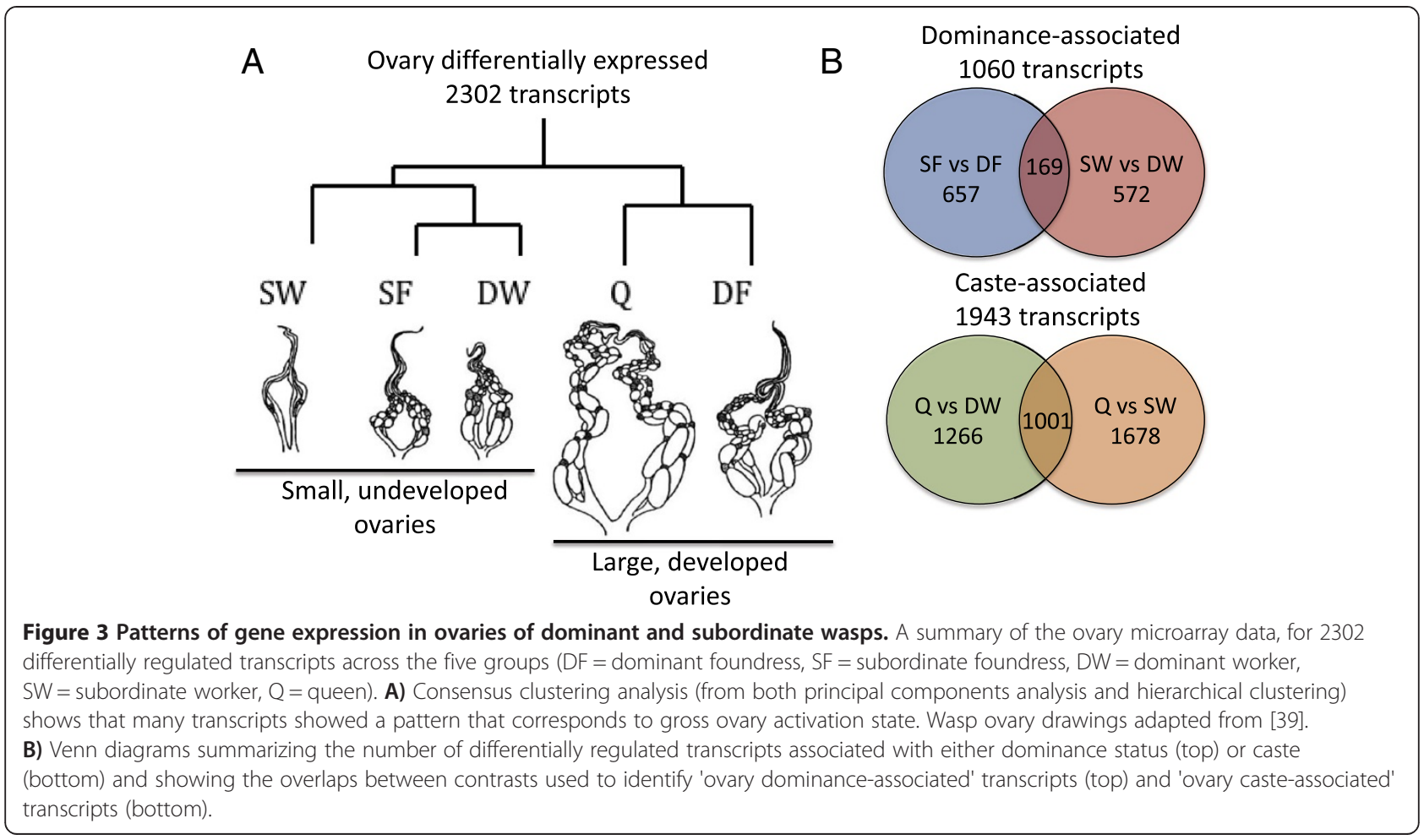

of which overlapped between the two contrasts (Figure 3B). The 1943 transcripts showing differences between queens and workers (the union of the aforementioned two contrasts) heretofore referred to as "ovary caste-associated" transcripts (Additional file 3).

\section{Gene ontology (GO) analysis on dominance and caste- associated gene lists}

Using DAVID [40], we tested to see which, if any, Gene Ontology categories (restricted to "Biological Process") of genes were under or over-represented in our gene lists compared the background array. The 60 "brain dominance-associated" transcripts were represented by several small clusters of genes (shown in Table 2), none of which were significantly overrepresented in the gene lists relative to the background gene set on the array: eye development, reproduction, and cytoskeletal organization. For the "brain caste-associated" transcripts, only one GO category, oxidation reduction, was significantly overrepresented relative to the background on the array, and this was only significant with unadjusted p-values (Table 2). Other processes associated with, but not significantly overrepresented, in brain caste-associated genes included aging, synaptic transmission, and RNA processing.

In general, both "ovary dominance-associated" and "ovary caste-associated" genes showed functions related to protein folding, mitotic spindle organization, proteolysis, and metabolism (Table 2). For the "ovary casteassociated" list, there were a number of genes related to reproduction and ovary activation, though none of these were significantly enriched relative to the background. There was a cluster of 70 genes related to "reproductive process", which included Insulin Receptor Substrate, Sex lethal, Female sterile (2) ketel, Ecdysone induced protein $75 B$. Another cluster of six genes was related to "oocyte fate determination", and included capping protein alpha, armadillo, and notch.

\section{Comparative analysis}

To begin to identify conserved pathways associated with caste and dominance, we tested for overlap between our complete lists of differentially regulated transcripts in brain $(n=502)$ and lists of differentially regulated transcripts from several studies in other species (Table 3).

We found no significant overlap between wasp brain differentially expressed transcripts and those differentially expressed in honey bees in association with caste differences [41] or response to queen pheromone [9]. We did find a significant overlap between wasp brain differentially expressed gene lists and those associated with differences in worker foraging behavior in honey bees in one study [42], but this result was not confirmed in comparisons with a second study on the same behavior [43].

We next investigated whether there was significant overlap with genes relating to aggressive behavior in honey bees. Alaux et al. [43] examined honey bee aggressive behavior in several contexts-as it relates to 
Table 2 Summary of Gene Ontology (GO) Analysis of differentially expressed gene lists

\begin{tabular}{lll}
\hline Cluster Biological process of cluster & \# genes $\begin{array}{l}\text { \# Enriched } \\
\text { subcategories }\end{array}$ & Example Drosophila homologs \\
&
\end{tabular}

\section{Brain dominance-associated}

C1 Compound eye development, photoreceptor cell 4 differentiation

C2 Cytoskeletal organization, actin filament organization

C3 Phagocytosis, vesicle mediated transport

C4 Reproduction, oogenesis

C5 Nucleotide, ATP binding

C6 Zinc, ion, metal binding

\section{Brain caste-associated}

C1 Oxidation reduction

C2 Aging, determination of adult life span

C3 Cell cycle process, microtubule-based process, cytoskeletal organization

C4 Regulation of RNA metabolism

C5 RNA splicing, binding, processing

C6 Metamorphosis, morphogenesis, cell death

C7 Synaptic transmission

\section{Ovary dominance-associated}

C1 Protein folding

C2 Proteolysis

C3 Mitotic spindle organization

C4 Oxidative phosphorylation

C5 Regulation of cell projection, morphogenesis, differentiation

C6 Carboxylic and amino acid catabolic process

C7 Lipoprotein metabolism

\section{Ovary caste-associated}

C1 Cytoskeletal organization, mitotic spindle organization

C2 Protein folding oxidative phosphorylation
COP9 complex homolog subunit 4, microtubule star, rasputin, scabrous

Paramyosin, Transitional endoplasmic reticulum, upheld

Beadex, alpha-coatomer protein

COP9 complex homolog subunit, Glutamate dehydrogenase, quick-to-court

Hexokinase A, polyA-binding protein, rasputin

Nucleosome remodeling factor - 38kD, Sorbitol dehydrogenase2, upheld

Ecdysone-induced protein 28/29kD, Glutathione peroxidase, Malate dehydrogenase, Sorbitol dehydrogenase-2

Autophagy-specific gene 7, Excitatory amino acid transporter 1

Eukaryotic initiation factor 4E, Helicase at 25E, Ribosomal protein L3, microtubule star, stubarista

Brahma associated protein $60 k D, X$ box binding protein- 1

Polyadenylate-binding protein 2, U2 small nuclear riboprotein auxiliary factor 50, hiiragi

Autophagy-specific gene 7, mastermind, scabrous

Glutamic acid decarboxylase, longitudinals lacking

Cyclophilin 1, DnaJ-like-2, Heat shock protein cognate 4, T-complex Chaperonin 5

Serine protease inhibitor 4, amontillado, supernumerary limbs, Proteasome $29 \mathrm{kD}$ subunit

Replication Protein A 70, Ribosomal protein S4, short spindle 4, Dynein heavy chain 64C

ATP synthase-beta, V-type proton ATPase subunit d 1 NADH:ubiquinone reductase $75 \mathrm{kD}$ subunit precursor

Calcium/calmodulin-dependent protein kinase II, twinstar, short stop, capping protein alpha

Glutamate dehydrogenase, Probable maleylacetoacetate isomerase 2, sluggish A

N-myristoyl transferase, Putative GPI-anchor transamidase, Rab escort protein

Brahma associated protein 55kD, Dynamitin, Kinesin heavy chain, notch

Calreticulin, Cyclophilin, Probable prefoldin subunit 4, Protein disulfide isomerase

Elongation factor 1-gamma, Transcription factor $\| B$, Ribosomal protein $\mathrm{S} 17$

Coenzyme Q biosynthesis protein 2, maroon-like, Succinate dehydrogenase B, Glutamate dehydrogenase

Aconitase, Aldolase, Cytochrome c oxidase subunit Va, Pyruvate kinase 
Table 2 Summary of Gene Ontology (GO) Analysis of differentially expressed gene lists (Continued)

\begin{tabular}{llll}
\hline C6 Proteolysis & 83 & $8 / 0$ & $\begin{array}{l}\text { Diphenol oxidase A2, Insulin degrading metalloproteinase, } \\
\text { Ubiquitin carrier protein, fizzy } \\
\text { C7 Glucose and hexose metabolism }\end{array}$ \\
Hexphase A, Phosphoenolpyruvate carboxykinase, \\
Phosphofructokinase
\end{tabular}

Based on a GO analysis using Drosophila homologs, the top 6 or 7 clusters of GO terms corresponding to "Biological Process" are shown. Each cluster listed is accompanied by a description of the GO terms that make up the "Biological Process of Cluster", "\# genes" represented in each cluster, "\# Enriched subcategories" which incidates GO subcategories that were significant within each cluster (counts refer to number of significant $p$-values, raw/FDR adjusted), and "Example Drosophila homologs" in each cluster.

genotype (aggressive Africanized lineages compared to more docile European lineages), age (hive bees vs foragers), and response to alarm pheromone (which elicits attack and stinging behavior). We first focused on the subset of genes that were found to be differentially expressed in all three contexts in honey bees. We found a small, but significant overlap between this list of genes and the complete set of brain differentially expressed genes in wasps. This suggestive result, along with the small size of the gene lists being compared, led us to compile an expanded list of all genes related to honey bee aggressive behavior in any context (the union of the three contexts). Here we found no significant overlap with the wasp brain differentially expressed list; however, when we compared this expanded honey bee aggression list to the wasp "brain caste-associated" genes, we again found significant overlap (Table 3 ). There was no significant overlap when we compared to the 63 wasp "brain dominance-associated genes" (data not shown).

To further investigate the potential connection between genes related to dominance (our study) and aggression, we compared our complete wasp brain differentially expressed gene list to microarray studies identifying brainexpressed genes associated with aggression in Drosophila melanogaster fruit flies [44] and maternal aggression in mice [45]. In both cases, we found evidence of a relatively small, but statistically significant overlap (Table 3). As a control, we compared our study to another mouse study that used the same microarray to examine brain gene expression patterns associated with sleep [46]—no significant overlap was detected.

\section{Discussion}

This study is the first comprehensive examination of chemical profiles and genome-wide expression patterns associated with reproductive dominance in a primitively eusocial species. Our analysis of cuticular hydrocarbons identified over a dozen compounds with potential links to the phase of the colony cycle (which encompasses season and social environment) in P. metricus. In addition, we provide new baseline data on transcriptomic correlates of reproductive dominance and caste in both brains and ovaries. Many genes showed expression patterns related to the social environment/season (founding phase vs worker phase, Figure 2), suggesting there could be major effects of social environment on brain gene expression in wasps. Thus, both the chemistry and brain transcriptome data show patterns strongly associated with the social environment, and highlight the fact that there are major differences in the social milieu between founding and worker phase colonies. These data agree with other recent studies suggesting the social environment as one of the most potent influences on gene expression patterns in ants [13]. Finally, our results indicate the brain expression patterns

Table 3 Comparative analyses examining overlap in gene expression between $P$. metricus, Apis, Drosophila, and Mus

\begin{tabular}{lllllllll}
\hline Wasp list & Compared to $\mathbf{X}$ & Description of study & Citation & Sig. both & Sig. wasp only & Sig. X only & Sig. neither & p-value \\
\hline Brain DE & Apis mellifera & Queen vs sterile worker & {$[41]$} & 77 & 129 & 354 & 498 & 0.322 \\
Brain DE & Apis mellifera & Queen phero. response & {$[9]$} & 58 & 339 & 384 & 2263 & 0.939 \\
Brain DE & Apis mellifera & Foragers vs nurses & {$[42]$} & $\mathbf{1 0 6}$ & $\mathbf{9 9}$ & $\mathbf{3 9 2}$ & $\mathbf{4 8 7}$ & $<0.001$ \\
Brain DE & Apis mellifera & Foragers vs nurses & {$[43]$} & 58 & 340 & 254 & 1478 & 0.99 \\
Brain DE & Apis mellifera & Aggression (composite) & {$[43]$} & 85 & 312 & 512 & 2135 \\
Brain DE & Apis mellifera & Aggression (3 contexts) & {$[43]$} & $\mathbf{5}$ & $\mathbf{8}$ & $\mathbf{3 9 2}$ & $\mathbf{2 6 4 0}$ & $\mathbf{0 . 0 1 9}$ \\
Brain Caste & Apis mellifera & Aggression (composite) & {$[43]$} & $\mathbf{3 7}$ & $\mathbf{8 2}$ & $\mathbf{4 3 5}$ & $\mathbf{1 5 7 5}$ \\
Brain DE & D. melanogaster & Aggression & {$[\mathbf{4 4}]$} & $\mathbf{4 9}$ & $\mathbf{3 0 7}$ & $\mathbf{2 3 8}$ & $\mathbf{2 2 4 7}$ & $\mathbf{0 . 0 2 2}$ \\
Brain DE & Mus musculus & Maternal aggression & {$[45]$} & $\mathbf{2 7}$ & $\mathbf{2 0 0}$ & $\mathbf{7 7}$ & $\mathbf{9 6 1 8 4}$ \\
Brain DE & Mus musculus & Sleeping vs awake & {$[46]$} & 68 & 159 & 323 & 714 & $\mathbf{0 . 0 3 3}$
\end{tabular}

Brain DE refers to the complete list of transcripts differentially expressed in the brain, "Brain Caste" refers to the subset of genes that are "brain caste-associated", described in the main text. The number of transcripts overlapping (significant in both, or "Sig. both"), as well as non-overlapping transcripts (significant in wasp only, or "Sig. wasp only"; significant in the other species, "Sig. X only"; and significant in neither study, "Sig. neither") and two-tailed p-values from Fisher's Exact Tests are shown. Lists of genes with significant overlaps are highlighted in bold and a complete list of overlapping transcripts are provided in Additional file 2. 
associated with reproductive dominance are (surprisingly) not conserved across wasps and honey bees, but rather that some genes associated with aggressive behaviors may have been co-opted to establish or maintain dominance hierarchies in Polistes wasps.

Previous studies have clearly demonstrated cuticular hydrocarbons change with female fertility in insects including Polistes, with some evidence for cuticular differences related to dominance status in foundresses/workers of other species of Polistes wasps [7,34,35,47,48]. We identified 13 cuticular hydrocarbons in $P$. metricus with significant differences across females. Several of these compounds have been identified on the cuticles of other insects, for example, in association with age in mosquitoes (pentacosane [49]), ovarian activation in social insects (dimethylpentatriacontane and dimethylhentriacontane [31], pentacosane, nonacosane, methylnonacosane, triacontane, and methyltriacontane [50]), and even dominance status in other species of Polistes (pentacosane, nonacosane, and methylnonacosane [32], methylpentatriacontane [51], and dimethylpentatriacontane [31]). However, in our study, none of these compounds were closely related to dominance status or levels of ovarian activation in P. metricus, but several showed associations with the time of collection (early vs late season) and/or social environment (foundress association vs queenright mature colony, Figure 1B). Further studies on these 13 compounds could provide additional insights into the role of cuticular hydrocarbons in response to the abiotic and social environment in Polistes.

Our microarray results suggest a relatively small subset of genes in the brain show patterns related to reproductive dominance status. There were several differentially expressed genes related to vision and eye development, which is intriguing because of the importance of visual communication in the genus Polistes, although $P$. metricus is not known to use visual cues for individual recognition $[52,53]$. We found no overlap between sets of differentially expressed genes between dominant and subordinate foundresses and dominant and subordinate workers, suggesting that distinct subsets of genes may be involved in the maintenance of dominance status in the founding and worker stages of colony development. This is consistent with known differences in the role of juvenile hormone $(\mathrm{JH})$ in the development and maintenance of dominance status and ovarian activation in queens versus workers in Polistes. In foundresses, $\mathrm{JH}$ regulates behavioral and ovarian reproductive dominance $[22,24,30]$. In workers, JH has a dual function in that it affects both reproductive dominance [20] and age-related onset of foraging behavior $[54,55]$, and $\mathrm{JH}$ action depends on the physiological condition (i.e. nutritional state) of the female [56,57].

A slightly larger subset of genes showed caste-associated expression differences in the brain. These genes had functions related to oxidation reduction, aging, and synaptic transmission, which could be linked to known differences in metabolism [58], lifespan [12], and learning abilities [59] between workers and queens. Previous studies in honey bees have also uncovered differences in the expression of genes related to aging (such as telomerase) and oxidation reduction [41]. We suggest candidate genes related to aging (Autophagy-specific gene 7, Excitatory amino acid transporter 1), eye development (microtubule star, rasputin, scabrous), and reproduction (Glutamate dehydrogenase, quick-to-court) may play an important role in establishing and maintaining adult caste differences in Polistes.

Our cross-species comparative analyses showed no significant overlap in sets of genes associated with dominance status in wasps and pheromonal regulation in honey bees. Thus, they do not support the hypothesis that pheromonal regulation of reproduction relies on the same molecular mechanisms as physical dominance in these two species. Furthermore, according to the ovarian and reproductive groundplan hypotheses [60-62], genes involved in reproduction have been co-opted to play a role in queenworker caste differentiation and worker division of labor. However, in contrast to this theory, we find distinct brain gene expression patterns are associated with reproductive dominance hierarchies between dominant and subordinate co-foundresses and between dominant and subordinate workers, and dominance-associated genes differ between wasps and honey bees. Thus, there does not appear to be a conserved suite of genes regulating these processes in the brain. Interestingly, however, genes associated with dominance in Polistes significantly overlap with sets of genes associated with aggressive phenotypes in honey bees [43], Drosophila [44], and mice [45]. There was also some overlap with genes related to foraging in one [42] of two [43] previous honey bee studies. This overlap may reflect differences in aggressive behavior between honey bee foragers and non-foragers [43], or perhaps be explained by the fact that lower dominance status in wasps is typically associated with increased foraging behavior [12]. Overall, these data suggest that there may be a small number of genes with recurrent roles in aggressive behavior across diverse taxa. It is important to note, however, that the microarray only examined a subset of the genes in the paper wasp genome and was limited to transcripts showing significant homology to honey bee or other insect proteins. The role of novel genes or rapidly evolving genes in the regulation of dominance status in Polistes remains to be explored and is definitely worthy of further attention [63].

We found large differences in ovary gene expression, both associated with dominance status and with caste differences. Overall, many transcripts showed expression differences associated with gross differences in ovary size (Figure 3A). This pattern is reflected in the types of genes that were differentially expressed-there were numerous 
genes with functions related to cell division and proliferation, as well as production of nucleic acids and proteins. Thus, the large differences in ovary size across the groups (Figure $3 \mathrm{~A}$ ) are undoubtedly produced by changes in the regulation of genes related to egg production and maturation.

\section{Conclusions}

In summary, experiments presented here provide a wealth of new data about the chemical and transcriptomic correlates of reproductive dominance in Polistes paper wasps, an important model system for studying dominance behavior and the evolution of sociality [74]. Several specific compounds and genes are excellent candidate for future studies of their causal role in establishing and maintaining dominance. Our data also highlight the importance of the season and/or social environment in gene expression and cuticular hydrocarbon production, and suggest there are distinct mechanisms responsible for communicating and maintaining dominance among foundresses, between queens and workers, and among workers. Comparisons with honey bees suggest that largely different sets of genes are associated with social regulation of reproduction in honey bees and paper wasps. This is not entirely surprising, considering bees and social vespids diverged between 100-150 million years ago [6], and that the form of social control of reproduction (chemical vs physical) differs greatly between the two species.

The most notable finding from our cross-species comparisons is that genes that are differentially expressed in brains of dominant and subordinate wasps are likely to be associated with aggression in other species, from honey bees, to flies, to mice. Our data suggest that in primitively social wasps, social regulation of reproduction may be regulated by genes with deeply conserved functions associated with aggression in solitary insects and other taxa. Thus, our data have begun to unravel the evolution of the mechanistic underpinnings of reproductive inhibition in workers, and that in some cases this may be built on fundamental elements of solitary behavior, such as aggression.

\section{Methods}

\section{Wasps}

We collected Polistes metricus adult females at four field sites in central Illinois (USA): Vermilion River Observatory (Danville, IL, +40 $3^{\prime} 28^{\prime \prime},-87^{\circ} 33^{\prime} 42^{\prime \prime}$ ), Allerton Park (Monticello, IL, $+40^{\circ} 0^{\prime} 25^{\prime \prime},-88^{\circ} 38^{\prime} 58^{\prime \prime}$ ), Lake of the Woods (Mahomet, IL, $+40^{\circ} 12^{\prime} 6^{\prime \prime},-88^{\circ} 22^{\prime}$ $38^{\prime \prime}$ ), and Forest Glen (Westville, IL, $+40^{\circ} 0^{\prime} 46^{\prime \prime},-87^{\circ} 33^{\prime}$ $\left.55^{\prime \prime}\right)$. We collected wasps from undisturbed nests located in wooden nest boxes or on the eaves of buildings between 5:30-7:00 am to ensure that all wasps were present on the nest and to control for circadian effects on gene expression. We collected 23 wasps during the founding phase between May 14-17, 2008 from nests with 2-3 females (10 nests with two and one nest with three females). We observed each nest $>2$ times in the 3 weeks prior to collection to verify the presence of multiple foundresses. Populations of Polistes metricus in Illinois generally have few nests that are multiply founded ( 5\%, A.L.T, personal observation). We collected 116 wasps during the "worker phase" between July 27- August 1, 2008 from 20 nests with at least 2 workers and no males; males are indicative of colonies producing non-worker reproductive females. To remove wasps from their nests, we anaesthetized them with $\mathrm{CO}_{2}$ gas for $30 \mathrm{sec}$, then immediately freezekilled them on dry ice and stored them at $-80^{\circ} \mathrm{C}$ for further analysis.

\section{Dissections and determinations of reproductive dominance rank}

Each wasp was subjected to several dissections (Additional file 1: Figure S1). We removed legs on dry ice and stored them at $-80^{\circ} \mathrm{C}$ for microsatellite analysis (see below). We noted wing wear (presence or absence) as an indicator of foraging experience [64]. We thawed gasters in RNA-later ${ }^{\circ}$ (Qiagen, Valencia, CA), then dissected ovaries and scored ovary activation $(1=$ completely undeveloped, string-like ovarioles, 2 = slightly developed ovarioles with small bulges, 3 = partially developed ovarioles, with two or fewer fully developed oocytes, 4 = fully developed ovarioles, with three or more fully developed oocytes). We stored ovaries in RNA-later ${ }^{\circ}$ at $-80^{\circ} \mathrm{C}$ for RNA extractions. We dissected Dufour's glands and sternal glands (located based on descriptions in [35]) from gasters and stored the glands in $200 \mu \mathrm{L}$ diethyl ether. After dissection, we submerged each wasp's gaster in $1 \mathrm{~mL}$ pentane for 10 minutes to extract cuticular hydrocarbons. We freeze-dried heads for $60 \mathrm{~min}$ at $300 \mathrm{mT}$ Torr, dissected brains on dry ice, and stored brains at $-80^{\circ} \mathrm{C}$ for RNA extractions. From the same heads we dissected mandibular glands (based on descriptions in [35]) and placed them in $200 \mu \mathrm{L}$ diethyl ether.

\section{Choice of focal wasps for microarray and chemical analysis}

To reduce variation due to differences in ovary activation, caste, and relatedness, we focused on a subset of collected wasps ( $\mathrm{n}=8$ focal females per group) in each of five groups: $\mathrm{DF}=$ dominant foundress, $\mathrm{SF}=$ subordinate foundress, $\mathrm{Q}=$ queen, $\mathrm{DW}=$ dominant worker, $\mathrm{SW}=$ subordinate worker. Wasp relatedness was assessed using microsatellites (Additional file 1, complete data in Additional file 4); this information allowed us to identify and exclude wasps from nests with evidence of queen replacement, which can profoundly disrupt dominance hierarchies in Polistes [65]. We focused on foundress associations with exactly two females, inferred from microsatellites to be sisters (but due to limits on sample size 
we included two pairs of non-sisters). We chose pairs of workers from the same nest inferred to be sisters and the daughters of the resident queen. We used foundress pairs that had clear differences in reproductive dominance-the subordinate female had an ovary activation score of 1 and the dominant female a score of 4 . For worker phase nests, we identified queens as females with ovary activation scores of 4 and high levels of wing wear (acquired during the founding phase, [64]). We chose dominant workers as females with no wing wear and ovary scores of 2 or 3 , and subordinate workers as females with high levels of wing wear and ovary scores of 1 .

\section{Chemical analysis}

Methodology for analysis of the three glands (mandibular, Dufour's and sternal) are presented in Additional file 1. A $1 \mu \mathrm{L}$ sub-sample of each cuticular extract was injected into an Agilent 6890 GC System using an Agilent HP$5 \mathrm{MS}$ column $(30 \mathrm{~m}$ length $\times 0.25 \mathrm{~mm}$ diameter $\times 0.25 \mathrm{um}$ thickness) in splitless mode and a flame ionization detector. The temperature program was as follows: $150^{\circ} \mathrm{C}$ hold for 1 minute, ramp up $15^{\circ} \mathrm{C} /$ minute to $200^{\circ} \mathrm{C}$, ramp up $7.5^{\circ} \mathrm{C} /$ minute to $300^{\circ} \mathrm{C}$, hold 25 minutes. Data were quantitated using Agilent Chemstation and internal standards. Eluting compounds were identified by comparing retention times and spectra (GC-MS) with those of pure standards.

We chose a subset of representative cuticular samples for GC-MS analysis on a Waters GCT gc-tof-ms using a similar column in splitless mode at $1 \mathrm{~mL} /$ minute $\mathrm{He}$ flow. Injector $250^{\circ} \mathrm{C}$; program: $50^{\circ} \mathrm{C}$, hold 1 minute, $20^{\circ} \mathrm{C} /$ minute to $180^{\circ} \mathrm{C}, 3^{\circ} \mathrm{C} /$ minute to $320^{\circ} \mathrm{C}$, hold 15 minutes. The identifications of 18 hydrocarbons from C25 (MW 352) to C40 (MW 562) were confirmed by either spectral comparison with the NIST MS Search 2.0 mass spectral library or by running standards.

\section{Microarrays}

The P. metricus oligo microarrays [11] are comprised of 10,000 duplicate spots, representing 5000 different transcripts, corresponding to approximately 3248 different genes. We extracted each individual wasp's brain and ovary RNA using a PicoPure (Evrogen, Moscow, Russia) kit. We assessed total RNA quantity with a NanoDrop (Thermo Scientific, Waltham, MA) and quality with an Agilent Bioanalyzer (Agilent, Santa Clara, CA). We then subjected each RNA sample to T7 amplification (Invitrogen, Grand Island, NY) and labeled each independently with both Cy3 and Cy5 dyes (Invitrogen, Grand Island, NY). We then hybridized amplified, labeled RNA to each microarray using previously described methods [11]. We employed a complete loop design with a dye swap, such that each individual wasp appeared on two arrays $(8$ wasps per group, 5 groups, 40 arrays, for both brain and ovary microarray studies). We hybridized arrays for approximately 18 hours at $42^{\circ} \mathrm{C}$ in a Maui mixer (BioMicro Systems, Salt Lake City, UT), then washed and immediately scanned them, with saturation tolerance set at $0.10 \%$, using a GenePix scanner (Molecular Devices, Sunnyville, CA). We manually spot-checked all arrays to remove spots with irregular printing or dust on the array.

\section{Statistical analysis}

For the chemical analysis, we calculated absolute concentrations of each compound for each individual sample using the external standard. These values were logtransformed and used for mixed model ANOVAs in R v. 2.15.3 (R Core Development Team 2008), with group (the five female types) as a fixed effect and colony as a random effect. Although the compounds on the cuticle are unlikely to be truly independent from each other, this analysis was useful as it allowed us to assess differences among groups for each compound separately. We conducted pairwise comparisons and adjusted p-values for multiple testing using a Bonferroni correction. We also used the same values for linear discriminant analysis (LDA) in $\mathrm{R}$ and hierarchical clustering analysis by compound (for significantly different compounds only, single linkage clustering method) in Genesis [49].

We used SAS to analyze microarray data as in [66]. We removed data from spots with intensity levels lower than the median background level of 175 and log transformed and normalized data across arrays using the Lowess method. We removed spots that were missing from $>25 \%$ of the arrays. We used a mixed model ANOVA to test for differences in expression, with dye and array as random effects and group and spot as fixed effects. P-values were corrected for multiple testing using false discovery rate (FDR), likewise for all pairwise comparisons between the groups. We used an FDR p-value significance cutoff of 0.05 for brains and 0.01 for ovaries. A more stringent cutoff was used for ovaries because there were a considerably larger number of differentially expressed transcripts. The results were visualized using principal components analysis and hierarchical clustering in JMP (SAS Institute, Cary, NC). We generated a distance matrix based on the number of differentially regulated transcripts between each pairwise comparison of female type, which we used to conduct hierarchical clustering in R. We conducted Gene Ontology analysis in DAVID [40], using Drosophila best hits to the wasp transcripts (as in [11]), and using the full set of Drosophila best hits on the P. metricus array as a background list. We report results of overrepresentation tests, both raw and Benjamini adjusted p-values.

To conduct tests of cross-species overlap, for each species, we used tBLASTx of $P$. metricus transcripts against other species' databases. Honey bee and Drosophila hits were used as described previously, with e-value cutoffs of $1 \mathrm{e}^{-5}$ [67]. For the mouse Mus musculus, we used 
BLAST2GO [68] against the Ensembl database and best tBLASTx hits were identified, with e-value cutoffs of $1 \mathrm{e}^{-3}$. If a $P$. metricus transcript did not have a hit meeting this cutoff, it was not used in further analyses. This resulted in lists of putative orthologs between P. metricus transcripts and each query species database. Using data in online repositories (GEO and MIAME), we identified the putative orthologs that were present on both test arrays being compared ( $P$. metricus and either bee, fly, or mouse). We determined the overlap between gene lists and used two-tailed Fisher Exact tests to determine whether the number of genes that were shared in common between both species was significantly higher or lower than expected by chance, compared to a hypergeometric distribution.

We tested for overlap between transcripts differentially expressed in wasp brains and transcripts with expression patterns in the brains of honey bees that were significantly associated with: 1) caste differences in adult queens and workers [41]; 2) exposure of workers to queen mandibular pheromone verses a solvent control [9]; 3) behavioral state differences between foragers and nurses [42,69]; and 4) aggressive behavior in workers [43]. We also tested for overlap between lists of differentially regulated genes related to aggression in Drosophila [44] and the mouse Mus musculus [45]. Although we found numerous additional studies examining aggressive behavior in other species including a cichlid fish [70], chicken [71], human [72], and a songbird [73], we were unable to conduct meaningful overlap analyses because of the small number of genes that met both criteria of being differentially expressed and having homologous sequences in P. metricus.

\section{Availability of supporting data}

All microarray data and details of the experiment were deposited in the Array Express database www.ebi.ac.uk/ arrayexpress (ArrayExpress accession number E-MTAB2190 for brain data and E-MTAB-2191 for ovary data) in accordance with MIAME ("Minimum information about a microarray experiment") standards.

\section{Additional files}

Additional file 1: Contains supplementary figures, tables, methods, results, and discussion to accompany the main text.

Additional file 2: Contains a list of brain differentially expressed transcripts, including lists of which overlapped across the different cross-species comparisons.

Additional file 3: Contains a list of ovary differentially expressed transcripts.

Additional file 4: Contains microsatellite data.

\section{Abbreviations}

GC: Gas chromatography; GC-MS: Gas chromatography-mass spectrometry; RNA: Ribonucleic acid; LDA: Linear discriminant analysis; PCA: Principal components analysis; FDR: False discovery rate.
Competing interests

The authors declare that they have no competing interests.

\section{Authors' contributions}

ALT conceived of the experiment, conducted GC and microarray studies, analyzed the data, and wrote the paper. JFT conducted GC-MS analyses, analyzed GC-MS data, and helped to draft the manuscript. SR performed comparative analyses. RM conducted GC-MS analyses and analyzed GC-MS data. MTH performed microsatellite analyses and analyzed microsatellite data. CMG participated in the design and coordination of the study and helped to draft the manuscript. All authors read and approved the final manuscript.

\section{Acknowledgments}

We would like to thank Gene Robinson for providing supplies and support for the initial stages of this project, David Galbraith for assistance with microarray spotfinding, Hollis Woodard for help with dissections and RNA extractions, Jim Tumlinson, Katalin Böröczky, Ezra Schwartzberg, and Nate McCartney for assistance with GC, and members of the Toth lab for reviewing the manuscript. This work was supported by a USDA-AFRI Postdoctoral Fellowship to A.L.T., and an NSF CAREER award to C.M.G.

\section{Author details}

'Department of Ecology, Evolution, and Organismal Biology, lowa State University, Ames, IA 50011, USA. ²Department of Entomology, lowa State University, Ames, IA, USA. ${ }^{3}$ Department of Entomology, Center for Pollinator Research, Center for Chemical Ecology, The Pennsylvania State University, University Park, PA, USA. ${ }^{4}$ Proteomics and Mass Spectrometry Core Facility, Huck Institutes for the Life Sciences, The Pennsylvania State University, University Park, PA, USA. ${ }^{5}$ Department of Biology, Grand Valley State University, Allendale, MI, USA.

Received: 3 June 2013 Accepted: 14 January 2014

Published: 28 January 2014

\section{References}

1. Adkins-Regan E: Hormones and animal social behavior. Princeton: Princeton University Press; 2005.

2. Wilson EO: The insect societies. Cambridge, Mass: Belknap Press of Harvard University Press; 1971.

3. Kocher SD, Grozinger CM: Cooperation, conflict, and the evolution of queen pheromones. J Chem Ecol 2011, 37(11):1263-1275.

4. Ratnieks F, Foster K, Wenseleers T: Conflict resolution in insect societies. Annu Rev Entomol 2005, 51:581.

5. Toth AL, Robinson GE: Evo-devo and the evolution of social behavior. Trends Genet 2007, 23(7):334-341.

6. Pilgrim EM, Von Dohlen CD, Pitts JP: Molecular phylogenetics of Vespoidea indicate paraphyly of the superfamily and novel relationships of its component families and subfamilies. Zool Scr 2008, 37(5):539-560.

7. Dapporto L, Santini A, Dani FR, Turillazzi S: Workers of a polistes paper wasp detect the presence of their queen by chemical cues. Chem Senses 2007, 32(8):795-802.

8. Winston ML: The biology of the honey bee. Cambridge, Mass: Harvard University Press; 1987.

9. Grozinger CM, Sharabash NM, Whitfield CW, Robinson GE: Pheromonemediated gene expression in the honey bee brain. Proc Natl Acad Sci U S A 2003, 100:14519-14525.

10. Le Conte $\mathrm{YaH}$ A: Primer pheromones in social Hymenoptera. Annu Rev Entomol 2008, 53:523-542.

11. Toth AL, Varala K, Henshaw MT, Rodriguez-Zas SL, Hudson ME, Robinson GE: Brain transcriptomic analysis in paper wasps identifies genes associated with behaviour across social insect lineages. Proc R Soc Edinb Biol 2010, 277(1691):2139-2148.

12. Hunt JH: The evolution of social wasps. USA: Oxford University Press; 2007.

13. Manfredini F, Riba-Grognuz O, Wurm Y, Keller L, Shoemaker DD, Grozinger CM: Sociogenomics of cooperation and conflict during colony founding in the fire ant, Solenopsis invicta. PLoS Genet 2013, 9(8):e1003633.

14. Ross KG, Matthews RW: The Social biology of wasps. Ithaca: Comstock Pub Associates; 1991.

15. Pardi L: Dominance order in Polistes wasps. Physiol Zool 1948, 21:1-13. 
16. Gamboa GJ SJL, Panek LM: The disappearance of subordinate foundresses in paper wasps: eviction by nestmates or reproductive strategy? Can J Zool 1999, 12:1928-1933.

17. Hughes CRB MO, Strassmann JE: Queen succession in the social wasp, Polistes annularis. Ethology 1987, 76:124-132.

18. Miyano S: Worker reproduction and related behavior in orphan colonies of a Japanese paper wasp, Polistes jadwigae (Hymenoptera, Vespidae). J Ethol 1991, 9(2):135-146.

19. Reeve HK: Polistes. In The Social Biology of Wasps. Edited by Ross KG, Matthews RW. Ithaca, NY: Comstock Publishing Associates; 1991:99-148.

20. Tibbetts EAH ZY: The challenge hypothesis in an insect: juvenile hormone increases during reproductive conflict following queen loss in Polistes wasps. Am Nat 2010, 176(2):123-130.

21. West-Eberhard MJ: The social biology of Polistine wasps. In Miscellaneous Publications. Ann Arbor, Michigan: University of Michigan; 1969:1-101.

22. Tibbetts EA, Sheehan MJ: The effect of juvenile hormone on Polistes wasp fertility varies with cooperative behavior. Horm Behav 2012, 61(4):559-564.

23. Tibbetts EA, Izzo A, Tinghitella RM: Juvenile hormone titer and advertised quality are associated with timing of early spring activity in Polistes dominulus foundresses. Insect Soc 2011, 58(4):473-478

24. Tibbetts EA, Izzo A, Huang ZY: Behavioral and physiological factors associated with juvenile hormone in Polistes wasp foundresses. Behav Ecol Sociobiol 2011, 65(5):1123-1131.

25. Tibbetts EA, Huang $Z Y$ : The challenge hypothesis in an insect: juvenile jormone increases during reproductive conflict following queen loss in Polistes wasps. Am Nat 2010, 176(2):123-130.

26. Pardi L, Turillazzi S: Polistes - Analysis of a Society (Hymenoptera Vespidae). Monit Zool Ital 1985, 19(3):164-164.

27. Tibbetts EA, Dale J: Individual recognition: it is good to be different. Trends Ecol Evol 2007, 22(10):529-537.

28. Roseler PF, Roseler I, Strambi A: Role of ovaries and ecdysteroids in dominance hierarchy establishment among foundresses of the primitively social wasp, Polistes gallicus. Behav Ecol Sociobio/ 1985, 18(1):9-13.

29. Roseler PF, Roseler I, Strambi A: Studies of the dominance hierarchy in the paper wasp, Polistes gallicus (L) (Hymenoptera-Vespidae). Monit Zool Ital 1986, 20(3):283-290.

30. Roseler PF, Roseler I, Strambi A, Augier R: Influence of insect hormones on the establishment of dominance hierarchies among foundresses of the paper wasp, Polistes gallicus. Behav Ecol Sociobiol 1984, 15(2):133-142.

31. Sledge MF, Boscaro F, Turillazzi S: Cuticular hydrocarbons and reproductive status in the social wasp Polistes dominulus. Behav Ecol Sociobiol 2001, 49(5):401-409.

32. Tannure-Nascimento IC, Nascimento FS, Zucchi R: The look of royalty: visual and odour signals of reproductive status in a paper wasp. Proc $R$ Soc Edinb Biol 2008, 275:2555-2561.

33. Dapporto L, Bruschini C, Cervo R, Petrocelli I, Turillazzi S: Hydrocarbon rank signatures correlate with differential oophagy and dominance behaviour in Polistes dominulus foundresses. J Exp Biol 2010, 213(3):453-458.

34. Dapporto L, Theodora P, Spacchini C, Pieraccini G, Turillazzi S: Rank and epicuticular hydrocarbons in different populations of the paper wasp Polistes dominulus (Christ) (Hymenoptera, Vespidae). Insect Soc 2004, 51(3):279-286.

35. Jeanne RL: The evolution of exocrine gland function in wasps. In Natural History and Evolution of Paper-wasps. Edited by Turillazzi S, West-Eberhard MJ. New York: Oxford University Press; 1996:144-160.

36. Espelie K, Wenzel J, Chang G: Surface lipids of social wasp Polistes metricus say and its nest and nest pedicel and their relation to nestmate recognition. J Chem Ecol 1990, 16:2229-2241.

37. Hunt JH, Mutti NS, Havukainen H, Henshaw MT, Amdam GV: Development of an RNA interference tool, characterization of its target, and an ecological test of caste differentiation in the eusocial wasp Polistes. PLoS One 2011, 6(11):e26641.

38. Toth AL, Varala K, Newman TC, Miguez FE, Hutchison SK, Willoughby DA, Simons JF, Egholm M, Hunt JH, Hudson ME, et al: Wasp gene expression supports an evolutionary link between maternal behavior and eusociality. Science 2007, 318(5849):441-444

39. Gobbi N, Noll F, Penna M: "Winter" aggregations, colony cycle, and seasonal phenotypic change in the paper wasp Polistes versicolor in subtropical Brazil. Naturwissenschaften 2006, 93(10):487-494.

40. Huang DW, Sherman BT, Lempicki RA: Systematic and integrative analysis of large gene lists using DAVID bioinformatics resources. Nat Protoc 2009, 4(1):44-57.
41. Grozinger CM, Fan YL, Hoover SER, Winston ML: Genome-wide analysis reveals differences in brain gene expression patterns associated with caste and reproductive status in honey bees (Apis mellifera). Mol Ecol 2007, 16(22):4837-4848.

42. Whitfield CW, Cziko AM, Robinson GE: Gene expression profiles in the brain predict behavior in individual honey bees. Science 2003, 302(5643):296-299.

43. Alaux C, Sinha S, Hasadsri L, Hunt GJ, Guzman-Novoa E, DeGrandi-Hoffman G, Uribe-Rubio JL, Southey BR, Rodriguez-Zas S, Robinson GE: Honey bee aggression supports a link between gene regulation and behavioral evolution. Proc Natl Acad Sci U S A 2009, 106(36):15400-15405.

44. Edwards AC, Rollmann SM, Morgan TJ, Mackay TF: Quantitative genomics of aggressive behavior in Drosophila melanogaster. PLoS Genet 2006, 2(9):e154

45. Gammie SC, Auger AP, Jessen HM, Vanzo RJ, Awad TA, Stevenson SA: Altered gene expression in mice selected for high maternal aggression. Genes Brain Behav 2007, 6(5):432-443.

46. Mackiewicz MNN, Zimmerman J, Pack A: Molecular mechanisms of sleep and wakefulness. Physiol Genomics 2007, 31(3):441-457.

47. Dapporto L, Sledge FW, Turillazzi S: Dynamics of cuticular chemical profiles of Polistes dominulus workers in orphaned nests (Hymenoptera, Vespidae). J Insect Physiol 2005, 51(9):969-973.

48. Lorenzi MC, Bagneres AG, Clement JL, Turillazzi S: Polistes biglumis bimaculatus epicuticular hydrocarbons and nestmate recognition (Hymenoptera, Vespidae). Insect Soc 1997, 44(2):123-138.

49. Sturn S, Quackenbush J, Trajanoski Z: Genesis: cluster analysis of microarray data. Bioinformatics Appl Note 2002, 18(1):207-208.

50. Smith AA, Millar JC, Hanks LM, Suarez AV: Experimental evidence that workers recognize reproductives through cuticular hydrocarbons in the ant Odontomachus brunneus. Behav Ecol Sociobiol 2012, 66:1267-1276.

51. Bonavita-Cougourdan A, Theraulaz G, Bagneres A-G, Roux M, Pratte M, Provost E, Clement J-L: Cuticular hydrocarbons, social organization and ovarian development in a Polistine wasp, Polistes dominulus Christ. Comp Biochem Physiol 1991, 100B(4):667-680

52. Gronenberg W, Ash LE, Tibbetts EA: Correlation between facial pattern recognition and brain composition in paper wasps. Brain Behav Evol 2008, 71(1):1-14.

53. Sheehan MJ, Tibbetts EA: Specialized face learning is associated with individual recognition in paper wasps. Science 2011, 334(6060):1272-1275.

54. Shorter JR, Tibbetts EA: The effect of juvenile hormone on temporal polyethism in the paper wasp Polistes dominulus. Insect Soc 2009, 56(1):7-13.

55. Giray T, Giovanetti M, West-Eberhard MJ: Juvenile hormone, reproduction, and worker behavior in the neotropical social wasp Polistes canadensis. Proc Natl Acad Sci U S A 2005, 102(9):3330-3335.

56. Tibbetts EAI AS: Endocrine mediated phenotypic plasticity: Conditiondependent effects of juvenile hormone on dominance and fertility of wasp queens. Horm Behav 2009, 56(5):527-531.

57. Tibbetts EAL S, Donajkowski K: Reproductive plasticity in Polistes paper wasp workers and the evolutionary origins of sociality. J Insect Physiol 2011, 57(7):995-999.

58. Weiner SA, Woods WA Jr, Starks PT: The energetic costs of stereotyped behavior in the paper wasp, Polistes dominulus. Naturwissenschaften 2009, 96(2):297-302.

59. Molina Y, O'Donnell S: Mushroom body volume is related to social aggression and ovary development in the paperwasp Polistes instabilis. Brain Behav Evol 2007, 70(2):137-144.

60. Amdam GV, Csondes A, Fondrk MK, Page RE Jr: Complex social behaviour derived from maternal reproductive traits. Nature 2006, 439(7072):76-78.

61. Amdam GV, Norberg K, Fondrk MK, Page RE Jr: Reproductive ground plan may mediate colony-level selection effects on individual foraging behavior in honey bees. Proc Natl Acad Sci U S A 2004, 101(31):11350-11355.

62. West-Eberhard MJ: Wasp societies as microcosms for the study of development and evolution. In Natural History and Evolution of Paper Wasps. Edited by West-Eberhard MJ, Turillazzi S. New York: Oxford University Press; 1996:290-317.

63. Ferreira PGP S, Chauhan R, Ffrench-Constant R, Gabaldón TG R, Sumner S: Transcriptome analyses of primitively eusocial wasps reveal novel insights into the evolution of sociality and the origin of alternative phenotypes. Genome Biol 2013, 14:R20.

64. Toth AL, Bilof KBJ, Henshaw MT, Hunt JH, Robinson GE: Lipid stores, ovary development, and brain gene expression in Polistes metricus females. Insect Soc 2009, 56(1):77-84. 
65. Strassmann JE, Fortunato A, Cervo R, Turillazzi S, Damon JM, Queller DC: The cost of queen loss in the social wasp Polistes dominulus (Hymenoptera : Vespidae). J Kansas Entomol Soc 2004, 77(4):343-355.

66. Kocher SD, Ayroles JF, Stone EA, Grozinger CM: Individual variation in pheromone response correlates with reproductive traits and brain gene expression in worker honey bees. PLoS One 2010, 5(2):e9116.

67. Toth AL, Varala K, Henshaw MT, Rodriguez-Zas SL, Hudson ME, Robinson GE: Brain transcriptomic analysis in paper wasps identifies genes associated with behaviour across social insect lineages. Proc Biol Sci 2010, 277(1691):2139-2148.

68. Conesa A, Götz S, García-Gómez J, Terol J, Talon M, Robles M: Blast2GO: a universal tool for annotation, visualization and analysis in functional genomics research. Bioinformatics 2005, 21(18):3674-3676.

69. Whitfield CW, Ben-Shahar Y, Brillet C, Leoncini I, Crauser D, Leconte Y, Rodriguez-Zas S, Robinson GE: Genomic dissection of behavioral maturation in the honey bee. Proc Natl Acad Sci U S A 2006, 103(44):16068-16075.

70. Renn S, Aubin-Horth N, Hofmann H: Fish and chips: functional genomics of social plasticity in an African cichlid fish. J Exp Biol 2008, 211:3041-3056.

71. Buitenhuis BHJ, Janss L, Sørensen P: Differentially expressed genes for aggressive pecking behaviour in laying hens. BMC Genomics 2009, 10:544.

72. Tochigi M, Iwamoto K, Kazuya, Bundo M, Miki, Sasaki T, Tsukasa, Kato N, Nobumasa, Kato T, Tadafumi: Gene expression profiling of major depression and suicide in the prefrontal cortex of postmortem brains. Neurosci Res 2008, 60(2):184-191.

73. Mukai MRK, Drnevich J, Wang G, Wacker D, et al: Seasonal differences of gene expression profiles in song sparrow (Melospiza melodia) hypothalamus in relation to territorial aggression. PLoS One 2009, 4(12):e8182.

74. Jandt JM, Tibbetts EA, Toth AL: Polistes paper wasps: a model genus for the study of social dominance hierarchies. Insectes Sociaux 2014, 61(1): $11-27$

doi:10.1186/1471-2164-15-75

Cite this article as: Toth et al: Shared genes related to aggression, rather than chemical communication, are associated with reproductive dominance in paper wasps (Polistes metricus). BMC Genomics 2014 15:75.

\section{Submit your next manuscript to BioMed Central and take full advantage of:}

- Convenient online submission

- Thorough peer review

- No space constraints or color figure charges

- Immediate publication on acceptance

- Inclusion in PubMed, CAS, Scopus and Google Scholar

- Research which is freely available for redistribution 